Bio - grafía. Escritos sobre la Biología y su Enseñanza. ISSN 2027-1034

Edición Extraordinaria. p.p. 1279 - 1287

Memorias del IX Encuentro Nacional de Experiencias en Enseñanza de la Biología y la Educación Ambiental. IV Congreso Nacional de Investigación en Enseñanza de la Biología.

\title{
ARTICULACIÓN DE ESTILOS DE APRENDIZAJE Y RUTINAS DE PENSAMIENTO PARAEL DESARROLLO DE COMPETENCIAS CIENTÍFICAS EN ESTUDIANTES DE SECUNDARIA
}

\author{
Narda Carolina Ascencio Peñaranda ${ }^{1}$ \\ Carlos Humberto Barreto Tovar ${ }^{2}$
}

\section{RESUMEN}

Al observar y analizar evidencias institucionales y de aula se identificó que los niños del grado séptimo del Colegio Brasilia Usme IED JT presentan múltiples estilos de aprendizaje y un nivel de desarrollo bajo en la competencia científica explicación de fenómenos, visibilizado a través de los niveles de sus argumentos.

Por tanto, se caracterizaron los estilos de aprendizaje de cada niño y a través de la metodología de Investigación - Acción (IA), se desarrollaron ciclos de reflexión - acción realizando contrastación teórica e identificando los movimientos de pensamiento que promueven dicha competencia, planteando y desarrollando estrategias que permitieran movilizar su desarrollo. Con base en ello, se propusieron actividades que se articularon en estrategias didácticas que utilizaron rutinas de pensamiento y a través de las cuales se han observado algunos cambios en los niveles de argumentación de algunos niños.

PALABRAS CLAVES: Competencia científica, explicación de fenómenos, rutinas de pensamiento, argumentación.

\section{ABSTRACT}

Observing and analyzing institutional and classroom evidence, it was identified that the seventh graders of the Brasilia Usme IED JT College present multiple learning styles and a low level of development in the scientific competence explanation of phenomena, visible through the levels of their Arguments.

Therefore, the learning styles of each child were characterized and through the methodology of Research - Action (AI), cycles of reflection - action were developed, performing theoretical contrast and identifying the movements of thought that promote this competence, proposing and developing strategies That allowed to mobilize its development. Based on this, activities were proposed that were articulated in didactic strategies that used routines of thought and through which some changes in the levels of argumentation of some children have been observed.

\footnotetext{
${ }^{1}$ Universidad de La Sabana. Facultad de Educación. Maestría en Pedagogía. Correo: nardaaspe@unisabana.edu.co

${ }^{2}$ Universidad de La Sabana. Facultad de Educación. Maestría en Pedagogía. Correo: carlos.barreto2@unisabana.edu.co
} 
Bio - grafía. Escritos sobre la Biología y su Enseñanza. ISSN 2027-1034

Edición Extraordinaria. p.p. 1279 - 1287

Memorias del IX Encuentro Nacional de Experiencias en Enseñanza de la Biología y la

Educación Ambiental. IV Congreso Nacional de Investigación en Enseñanza de la Biología.

KEYWORDS: Scientific competence, explanation of phenomena, routines of thought, argumentation.

\section{INTRODUCCIÓN}

En el presente artículo se describen los resultados parciales de una investigación en la cual se articulan las rutinas de pensamiento con los estilos de aprendizaje de los estudiantes para promover el desarrollo de la competencia científica explicación de fenómenos visibilizado a través del nivel argumentativo de los estudiantes.

La formación en competencias científicas es una de las metas en la enseñanza de las ciencias naturales en Colombia, por tanto, debe ser considerada como una prioridad al momento de diseñar las estrategias de enseñanza que orienten el desarrollo de las clases. En ese sentido, se espera que, en el transcurso de su vida escolar, los estudiantes desarrollen competencias científicas que les permita proponer y ejecutar acciones a través de las cuales den respuesta a problemáticas prácticas o teóricas desde el área de ciencias naturales.

Con base en la lectura de diversos autores, Organización para la cooperación y desarrollo económico OCDE (2006); Arteta, Fonseca, lbañez, Chona y Martínez (2009) y Quintanilla, M.; C. Joglar; R. Jara; J. Camacho; E. Ravanal; A. Labarrere; L. Cuellar; M. Izquierdo; J. Chamizo. (2010), para la presente investigación se entiende por competencia científica el conjunto de saberes y habilidades que permiten a los estudiantes conocer, comprender y explicar los fenómenos naturales y del mismo modo proponer soluciones a situaciones problémicas teóricas y de su vida cotidiana con base en procedimientos y saberes propios de la ciencia; las competencias científicas se traducen en desempeños que son observables y evaluables y se desarrollan en contextos específicos.

En Colombia, una de estas competencias es la explicación de fenómenos que es definida por el Instituto Colombiano para el fomento de la Educación Superior (ICFES, 2015) como "la capacidad de construir explicaciones y comprender argumentos y modelos que den razón de fenómenos, y de establecer la validez o coherencia de una afirmación o de un argumento relacionado con un fenómeno o problema científico" (p. 8). Por tanto, es posible inferir que para explicar un fenómeno se requiere identificar en el mismo sus variables, establecer los argumentos que soporten dicha explicación, organizarlos de forma lógica y consistente con las evidencias que se presenten y establecer conexiones tanto con conocimientos previos como con las evidencias aportadas. Estas explicaciones se pueden expresar como argumentos que dan cuenta de los fenómenos y en consecuencia exige organizar las ideas de modo tal que permitan establecer de forma consistente las razones que soportan tal o cual explicación.

Ahora bien, los argumentos construidos por un estudiante no son siempre de la misma calidad o presentan las mismas características, en ese sentido Erdurán et ál. (2004) y Erduran (2008), citados por Tamayo (2011) proponen que es posible evaluar la calidad de los argumentos con base en cinco niveles argumentativos que describen la compeljidad de los argumentos. La construcción de argumentos implica movilizar el pensamiento, es decir pensar para proponer explicaciones que den cuenta de un fenómeno. 
Bio - grafía. Escritos sobre la Biología y su Enseñanza. ISSN 2027-1034

Edición Extraordinaria. p.p. 1279 - 1287

Memorias del IX Encuentro Nacional de Experiencias en Enseñanza de la Biología y la

Educación Ambiental. IV Congreso Nacional de Investigación en Enseñanza de la Biología.

Aparecen como opción para movilizar el pensamiento las rutinas de pensamiento que son definidas por Ritchhart, et al (2014) como "procedimientos, procesos o patrones de acción que se utilizan de manera repetitiva para manejar o facilitar el logro de tareas específicas" por tanto se entiende que una rutina de pensamiento es un conjunto de acciones direccionadas hacia la consecución de una meta específica de comprensión y que en consecuencia movilizan uno o varios movimientos del pensamiento de forma intencionada y se realizan con relativa frecuencia en el aula de clase. En ese sentido De Castro Tovar (2012) afirma que las rutinas de pensamiento son herramientas utilizadas una y otra vez en el aula para apoyar un pensamiento específico, por ejemplo, hacer conexiones, describir, construir explicaciones, considerar diferentes puntos de vista y perspectivas, captar lo esencial, sacar conclusiones y razonar con evidencia y permiten el desarrollo de patrones adoptados para ayudarnos a formar pensamientos, razonar o reflexionar.

\section{METODOLOGÍA}

La investigación se desarrolló con 23 estudiantes del Colegio Brasilia Usme IED que durante el año 2016 cursaban grado séptimo y fueron promovidos a grado octavo en el año 2017. Este colegio se encuentra ubicado al sur de la ciudad de Bogotá D.C., en la localidad de Usme que corresponde al estrato socioeconómico 1 y 2. El grupo de estudiantes se caracterizó por presentar inicialmente un vocabulario limitado, descripciones imprecisas en las cuales solían confundir conceptos, los argumentos empleados para explicar fenómenos eran en su mayoría intuitivos, no se soportaban en evidencias u obedecían a sus observaciones primarias de los fenómenos, se evidenciaban serias dificultades tanto gramaticales como de redacción y un pobre desarrollo de la capacidad para discriminar.

La presente investigación se enmarca en el enfoque cualitativo ya que pretende realizar interpretaciones en torno a un fenómeno educativo particular, en este caso la incidencia de estrategias de enseñanza que articulen los estilos de aprendizaje en el desarrollo de la competencia explicación de fenómenos. Se adopta como diseño de investigación, la investigación acción por considerar que a través de ciclos de reflexión - acción se construiría conocimiento a propósito del problema planteado con la intención de modificar las prácticas pedagógicas de la docente y mejorar el nivel de los estudiantes en dicha competencia.

Los instrumentos empleados para la recolección de información fueron:

Prueba estandarizada - Index of Learning Styles - ILS: Esta prueba que fue referenciada por Ramírez Sánchez, P (2015) es un insumo útil para caracterizar las tendencias en cuanto a estilos de aprendizaje de acuerdo con lo propuesto por Felder y Soloman (1996).

Diarios de Campo: Se empleó para registrar las observaciones llevadas a cabo durante el proceso investigativo. Para el análisis de los datos registrados se establecieron pre-categorías a las cuales se asignó un color particular, a continuación, se resaltaban las unidades de análisis de acuerdo con el color de la pre-categoría a la cual eran asignados. 
Bio - grafía. Escritos sobre la Biología y su Enseñanza. ISSN 2027-1034

Edición Extraordinaria. p.p. 1279 - 1287

Memorias del IX Encuentro Nacional de Experiencias en Enseñanza de la Biología y la Educación Ambiental. IV Congreso Nacional de Investigación en Enseñanza de la Biología.

Producciones de los estudiantes: Para el presente informe se analizaron las producciones escritas de 23 estudiantes que cursaron grado séptimo (2016) y fueron promovidos a octavo (2017) para lo cual se asignó un código a cada estudiante y sus escritos se transcribieron y categorizaron. Su análisis se llevó a cabo a través del análisis de contenido semántico, el cual "pretende ante todo estudiar las relaciones entre temas tratados en un texto" Abela, J (2012).

La construcción de las categorías de investigación se realizó con base en la definición planteada por el lcfes (2015) sobre la competencia explicación de fenómenos y la tabla de niveles propuesta por Toro et al (2007), estableciendo además relaciones con los niveles de argumentación citados por Tamayo (2011). En la tabla 1 se presenta la asociación que se propone entre dichos elementos teóricos:

Tabla 1. Categorías de Explicación asociadas a argumentación

\begin{tabular}{|c|c|}
\hline BAJO & $\begin{array}{l}\text { E1A1. Identificar características, regularidades e } \\
\text { irregularidades en un evento o fenómeno a través de } \\
\text { argumentos que son descripciones simples. }\end{array}$ \\
\hline \multirow{2}{*}{$\begin{array}{l}\text { El estudiante da razones de } \\
\text { fenómenos y eventos tangibles y } \\
\text { cercanos poniendo en juego la } \\
\text { imaginación y el dominio de nociones } \\
\text { y relaciones lógicas sencillas. }\end{array}$} & $\begin{array}{l}\text { E1A2 Identificar características, regularidades e } \\
\text { irregularidades en un evento o fenómeno a través de } \\
\text { argumentos que presentan claramente datos y } \\
\text { conclusión. }\end{array}$ \\
\hline & $\begin{array}{l}\text { E1A3 Identificar características, regularidades e } \\
\text { irregularidades en un evento o fenómeno a través de } \\
\text { argumentos que presentan datos, conclusión y } \\
\text { justificación. Hacen uso de conectores. }\end{array}$ \\
\hline
\end{tabular}

\section{RESULT ADOS Y DISCUSIÓN}

Al aplicar Prueba estandarizada - Index of Learning Styles - ILS se obtuvieron los resultados que se presentan en la gráfica 1.

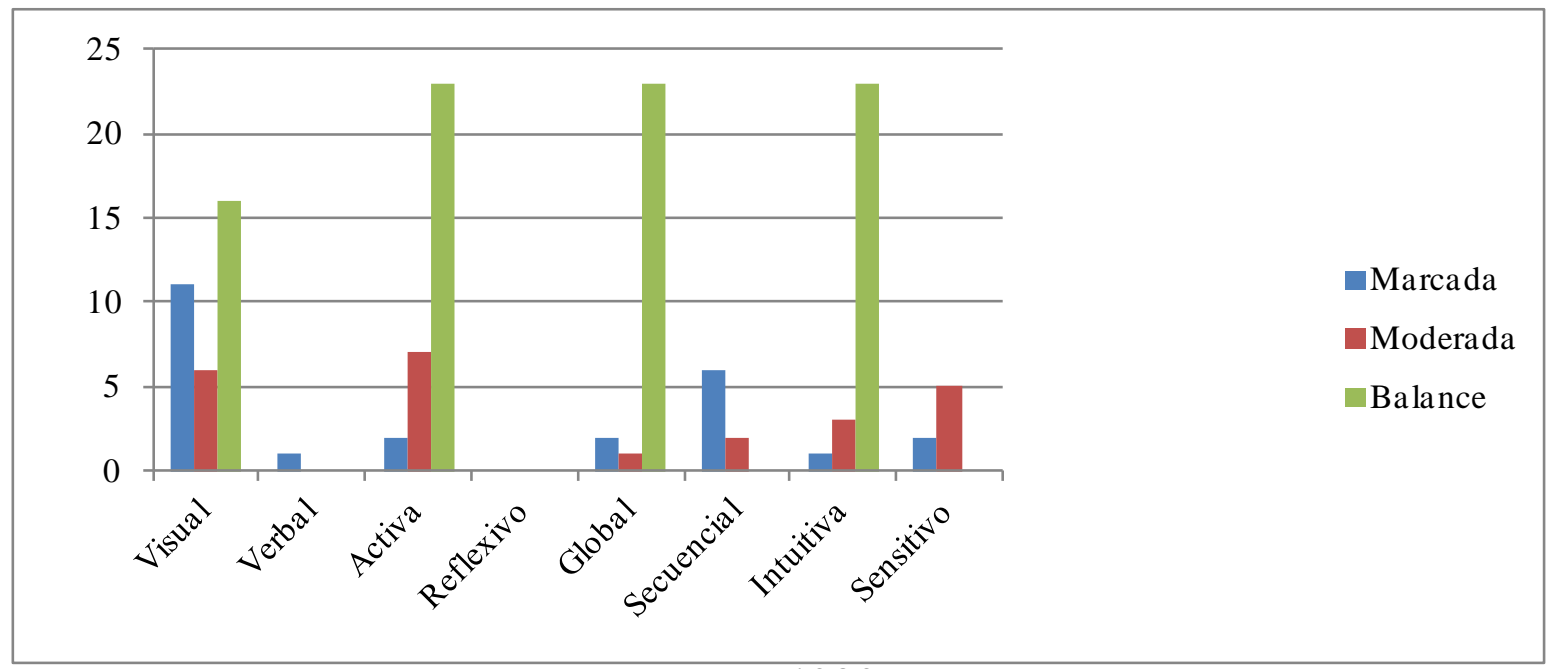

Grá fica 
Bio - grafía. Escritos sobre la Biología y su Enseñanza. ISSN 2027-1034

Edición Extraordinaria. p.p. 1279 - 1287

Memorias del IX Encuentro Nacional de Experiencias en Enseñanza de la Biología y la Educación Ambiental. IV Congreso Nacional de Investigación en Enseñanza de la Biología.

1. Síntesis estilos de aprendizaje.

De acuerdo con ello, se encontró que, en cuanto a la dimensión de representación, el $32 \%$ de los estudiantes presentan una tendencia marcada hacia el estilo visual y el $17,5 \%$ tendencia moderada hacia el mismo estilo; es decir el $49.5 \%$ de los estudiantes prefieren recibir la información a través de gráficos, películas, cuadros o diagramas de flujo y de ellos el $32 \%$ podrían presentar dificultades en ambientes de aprendizaje que no sean compatibles con esa preferencia. En la dimensión de procesamiento, el $20 \%$ presentan una tendencia marcada hacia el estilo activo por lo cual es probable que aprendan más fácilmente en un entorno de enseñanza que favorezca la manipulación directa de los objetos y el trabajo con otros. Con respecto a la dimensión de percepción, el $18 \%$ de los estudiantes presentan un tendencia marcada y moderada hacia el estilo sensitivo, son estudiantes que tienden a ser concretos, prácticos, guiados hacia los hechos y los procedimientos, tienen un alto nivel de energía para hacer cosas que son pragmáticas, lógicas y útiles.

Cabe resaltar que el $67 \%$ del grupo presenta tendencia en balance para las tres últimas dimensiones (gráfica 1) por tanto se esperaría que aprendieran con la misma facilidad en ambientes de aprendizaje que presenten actividades propias de cualquiera de los dos estilos para dichas dimensiones. En cuanto a la dimensión de comprensión, el 17.6\% presentan una tendencia moderada hacia el estilo secuencial, lo cual supone que son estudiantes que aprenden de lo particular a lo general, paso a paso y al no tener actividades que presenten estas características es probable que se dificulte su proceso de aprendizaje.

En consecuencia, se eligieron tres rutinas de pensamiento que movilizan el pensamiento deseado y que permiten vincular en ellas los estilos de aprendizaje con mayor tendencia en el grupo, se presenta en la tabla 2 una de ellas.

Tabla 2. RUTINAS DE PENSAMIENTO VS ESTILOS DE APRENDIZAJE

\begin{tabular}{|ll|}
\hline VISUAL & OBSERVO CONTRASTO EXPLICO \\
\hline ACTIVO & $\begin{array}{l}\text { Pomenta la observación detallada y genera curiosidad. } \\
\text { observar y el trabajo individual o colectivo }\end{array}$ \\
\hline SECUENCIAL & Implica desarrollar paso paso \\
\hline
\end{tabular}

Se llevaron a cabo tres ciclos de reflexión acción, en los cuales se analizaron las producciones escritas de los estudiantes. En la tabla 3 se presentan los resultados para el nivel bajo en los tres ciclos. 
Bio - grafía. Escritos sobre la Biología y su Enseñanza. ISSN 2027-1034

Edición Extraordinaria. p.p. $1279-1287$

Memorias del IX Encuentro Nacional de Experiencias en Enseñanza de la Biología y la Educación Ambiental. IV Congreso Nacional de Investigación en Enseñanza de la Biología.

Tabla 1 Frecuencia en las Unidades de Análisis

\begin{tabular}{|c|c|c|c|c|}
\hline NIVEL & Categorías & FUA ciclo 1 & FUA ciclo 2 & FUA ciclo 3 \\
\hline \multirow{3}{*}{ BAJO } & E1A1. & 28 & 11 & 5 \\
\cline { 2 - 5 } & E1A2 & 26 & 33 & 19 \\
\cline { 2 - 5 } & E1A3 & 0 & 11 & 1 \\
\hline
\end{tabular}

De acuerdo con el ICFES (2015) se espera que los estudiantes puedan explicar cómo ocurren algunos fenómenos de la naturaleza a partir de observaciones, patrones y conceptos propios del conocimiento científico. Para ello se requiere que logren identificar las características y las regularidades en el mismo y en consecuencia movilicen su pensamiento en términos de "Observar de cerca y describir que hay ahi" Ritchhart, et al (2014). Con base en los resultados durante el ciclo 1 para la categoría E1 A1, se encontró que los niños presentaron descripciones simples, sus observaciones fueron superficiales y utilizaron un vocabulario básico muchas veces impreciso:

"Veo un pelito con un cordon y muchos pelitos. Tierra, una mata y unas hojas con un tallo. Animales que vuelan, saltan y que caminan. Tres mujeres, tres hombres, personas. "CV

"veo un sistema con venas, corazon, Arterias y capilares en el ser humano y en el perro." $A C A D$

"Pues aprendi que las plantas recogian elementos organos y tejidos la cual heran los tallos raizes y hojas los organos y los elemento H2O y CO2". GLDA

"Algo feo con pelos. Tierra, hojas y da tallo. Yo veo 10 animales con diferentes tamaños. Yo veo unas personas. 3 hombres y 3 mujeres. Yo estoy viendo el organismo de un ser humano y de un animal." GRAF

Estas observaciones difícilmente pueden servir de base para construir explicaciones profundas de un fenómeno.

Durante el ciclo 2 aparecen datos ubicados en las categorías E1A1, E1A2 y E1A3. Disminuyó la frecuencia para la categoría E1A1 y aumentó la frecuencia para la categoría E1A3 ya que los argumentos que escribieron durante este ciclo se caracterizaron por presentar mayor extensión, emplear un vocabulario más adecuado y justificar las regularidades encontradas en la imágenes basándose en las observaciones hechas, esta situación se presentó probablemente porque las actividades desarrolladas exigían afinar el proceso de observación e identificación de regularidades, especialmente la actividad llamada "observo contrasto explico" ya que pretendía fortalecer la explicación a partir de observaciones que permitieran identificar las características y las regularidades en un fenómeno, situación que se evidencia en el siguiente fragmento de clase:

"En ese momento Juan Carlos llama a la profesora y le pregunta, profe yo observo que el ave tiene como más pulmones, porqué crees eso Juan Carlos, le pregunta la maestra y él contesta porque mire aquí en la imagen se ven como bolas a mí me parece que son pulmones." 15 
Bio - grafía. Escritos sobre la Biología y su Enseñanza. ISSN 2027-1034

Edición Extraordinaria. p.p. $1279-1287$

Memorias del IX Encuentro Nacional de Experiencias en Enseñanza de la Biología y la

Educación Ambiental. IV Congreso Nacional de Investigación en Enseñanza de la Biología.

Estas actividades fueron planteadas por presentar un fuerte componente visual, de modo tal que estaban acorde con la preferencia en términos de la vía sensorial a través de la cual la mayoría de los estudiantes en cuestión prefieren captar la información ya que Felder y Silverman (1998) afirman que los estudiantes visuales prefieren recibir la información a través de gráficos, películas, cuadros o diagramas de flujo. Por otro lado, se ha afirmado que el uso de las imágenes que "parecen tener un papel importante en el aprendizaje de las ciencias. Por ejemplo, es mucho más fácil construir un modelo mental a través de la percepción visual que a través del discurso" Moreira, Greca, \& Rodriguez, (2002, p. 20), así mismo Romero y Pulido (2015) indican que estas pueden contribuir a mejorar los aprendizajes en la enseñanza de las ciencias experimentales.

A continuación, se presentan algunos ejemplos de argumentos que corresponden a la categoría E1A3:

"Vemos 2 seres vivos con su respectivo sistema respiratorio. 1era imagen: vemos un animal con alas, patas, pico y plumas. Tiene su sistema respiratorio. 2da imagen: vemos una silueta de una persona con su sistema respiratorio. Se parecen en que los dos tienen sistema respiratorio. Los dos son seres vivos. Se diferencian El ave tiene mas de dos pulmones y el humano solo dos." CGMA

En este argumento se evidencia el esfuerzo por hacer una descripción más clara de lo observado, haciendo uso de un lenguaje más preciso probablemente porque se realizó una observación más detallada lo cual podría indicar que operó el movimiento de pensamiento de "Observar de cerca y describir que hay ahî" y además de ello se comunicó a los estudiantes los criterios de evaluación que se emplearían para la valorar sus trabajos.

"El sistema respiratorio del ave y del humano, los pulmones del humano tienen una forma muy parecida a un triangulo u ovalo y la del ave tiene una forma de pera deforme. Se diferencian porque uno es mucho mas diferente ya que los pulmones tienen distintas formas ya que en el hombre son dos y en el otro como seis."BMJC

En este argumento, además de lo señalado para el anterior, se presenta la justificación referida únicamente a lo observado en la imagen, no son evidentes relaciones, por ejemplo, adaptativas para dar cuenta de las diferencias o similitudes.

Durante el ciclo 3 aparecen datos ubicados en las categorías E1A1 y E1A2. Al igual que el ciclo 2, disminuye la frecuencia para la categoría E1A1 y aumenta la frecuencia para la categoría E1A2.

"Veo un señor comiendo comida chatarrra pienso que el señor se esta contaminando su cuerpo. Veo una señora alimentandose con ensalada pienso que esta comiendo algo saludable.E1A2" GLDA

"Veo una mujer con una ensalada, pienso que esta comiendo algo saludable.E1A2"GABS 
Bio - grafía. Escritos sobre la Biología y su Enseñanza. ISSN 2027-1034

Edición Extraordinaria. p.p. $1279-1287$

Memorias del IX Encuentro Nacional de Experiencias en Enseñanza de la Biología y la

Educación Ambiental. IV Congreso Nacional de Investigación en Enseñanza de la Biología.

En estas explicaciones se identifican claramente las variables presentadas, pero se limitan a presentar conclusiones, que si bien permiten intuir justificaciones latentes no se hacen visibles a través del argumento. Aparece un solo dato para la categoría E1A3 probablemente porque durante este ciclo no se realizaron actividades enfocadas a justificar regularidades presentes en los fenómenos ya que se dio más peso a otro tipo de ejercicios como la generación de propuestas frente a diferentes eventos.

\section{CONCLUSIONES}

La cantidad de estudiantes que se encontraban en la categoría A1E1 que corresponde al nivel bajo se disminuyó notablemente y aumentó la cantidad en nivel A1E2 y A1E3, que, aunque continúan correspondiendo al nivel bajo, presentan un mayor grado de complejidad argumentativa. Por tanto, se considera que el nivel de desarrollo de la competencia mejoró en los estudiantes.

Teniendo en cuenta que las competencias se traducen en desempeños que son observables y evaluables, se encuentra que un indicador útil del grado de desarrollo de la competencia explicación de fenómenos es el nivel argumentativo de los estudiantes.

Se confirma que como lo plantean Cabrera y Fariñas (2001), Ramírez Sánchez, P (2015) y Quintanal (2012) tener en cuenta los estilos de aprendizaje de los estudiantes para la planeación y ejecución de actividades en la clase, mejora su desempeño académico y el desarrollo de sus habilidades mentales.

En consecuencia, se pudo constatar que el planear las estrategias de clase a partir del reconocimiento de los estilos de aprendizaje se reflejó en la mejoría en cuanto a la calidad de los argumentos planteados por los estudiantes.

\section{BIBLIOGRAFÍA}

Abela, J. A. (2002). Las técnicas de análisis de contenido: una revisión actualizada. Recuperado de http://anthropostudio.com/wp-content/uploads/2014/07/Andr\%C3\%A9u-J.2000.-Las-t\%C3\%A9cnicas-de-an\%C3\%A1lisis-de-contenido-una-revisi\%C3\%B3nactualizada..pdf

Arteta, J.; Fonseca, G.; Ibáñez, S.; Chona, G. y Martínez, S. (2009). El conocimiento profesional del profesor y el desarrollo de competencias científicas investigativas. Enseñanza de las Ciencias, Número Extra VIII Congreso Internacional sobre Investigación en Didáctica de las Ciencias, Barcelona, pp. 3222-3227 Recuperado de http://ensciencias.uab.es/congreso09/numeroextra/art-3222-3227.pdf

Cabrera y Fariñas (2005) El estudio de los estilos de aprendizaje desde una perspectiva vigostkiana: una aproximación conceptual. Revista lberoamericana de Educación. No 37/1

De Castro Tovar, M (2012) Las rutinas de pensamiento: una estrategia para visibilizar mi aprendizaje. Universidad de la Sabana. Chía 
Bio - grafía. Escritos sobre la Biología y su Enseñanza. ISSN 2027-1034

Edición Extraordinaria. p.p. 1279 - 1287

Memorias del IX Encuentro Nacional de Experiencias en Enseñanza de la Biología y la Educación Ambiental. IV Congreso Nacional de Investigación en Enseñanza de la Biología.

Grateron F, G. (2014). Las estrategias metodológicas utilizadas por los maestros. Universidad de La Sabana. Chía, Colombia.

Felder, R y Soloman, B (1996) Index of learning styles (ils). Recuperado de http://www4.ncsu.edu/unity/lockers/users/f/felder/public/lLSdir/styles.htm

Instituto colombiano para la evaluación de la educación. Icfes (2015) Lineamientos generales para la presentación del examen de Estado SABER 11․ Bogotá: El autor.

Moreira, M., Greca, I., \& Rodriguez, L. (2002). Modelos mentales y modelos conceptuales en la enseñanza y aprendizaje de las ciencias. Revista Brasileira de Investigación en Educación en ciencias., 36-56.

Organización para la cooperación y el desarrollo económico. OCDE (2006) El programa PISA de la OCDE. Qué es y para qué sirve. Recuperado de https://www.oecd.org/pisa/39730818.pdf

Quintanal Pérez, F. (2012). Relación entre estilos de aprendizaje y rendimiento escolar en física y química de secundaria. Vivat Academia, 0(117E), 1143-1153. doi:10.15178/va.2011.117E.1143-115

Quintanilla, M.; C. Joglar; R. Jara; J. Camacho; E. Ravanal; A. Labarrere; L. Cuellar; M. Izquierdo; J. Chamizo. (2010) "Resolución de problemas científicos escolares y promoción de competencias de pensamiento científico. ¿Qué piensan los docentes de química en ejercicio?." Enseñanza de las ciencias: revista de investigación y experiencias didácticas [online], 2010, Vol. 28, Núm. 2, p. 185-198. http://www.raco.cat/index.php/Ensenanza/article/view/199612/353386 [Consulta: 20-1016]

Ramírez Sánchez, P (20015) Relación entre los estilos de aprendizaje y el desarrollo de habilidades de pensamiento - análisis y resolución de problemas - en el área de ciencias naturales y educación ambiental con estudiantes del ciclo quinto. Trabajo de grado para obtener el título de Magíster en Pedagogía. Universidad de La Sabana. Chía, Colombia.

Romero Rincón, y, Pulido Serrano, G (2015) Incidencia de las rutinas de pensamiento en el fortalecimiento de habilidades científicas: observar y preguntar en los estudiantes de grado cuarto, ciclo II del colegio rural José Celestino Mutis I.E.D. Trabajo de grado para obtener el título de Magíster en Pedagogía. Universidad de La Sabana. Chía, Colombia.

Ritchhart, R; Church, M; Morrison, K (2014). Hacer visible el pensamiento. Buenos Aires Argentina Ed. Paidós

Tamayo Alzate, O (2011) La argumentación como constituyente del pensamiento crítico en niños. HALLAZGOS. Año 9, No. 17. Bogotá, D.C: Universidad Santo Tomás / pp. 211233

Toro, J., Blandon, C., Martinez, R., Casteblanco, Y., Cardenas, F., \& Granez, J. (2007). Fundamentación Conceptual Área de Ciencias Naturales. Bogotá: ICFES. 